\title{
Influence of Working Memory Capacity on the Frequency of Self-Repairs
}

\author{
Lilei Gao* \\ Xi'an Kedagaoxin University, Xi'an 710109, China \\ *Corresponding author: Lilei Gao, $475899655 @ q q . c o m$
}

\begin{abstract}
This article explored the influence of working memory capacity on the frequency of self-repairs. The narrative task and listening span task were used. Twenty post-graduate students participated in this study. Overall, the results of this study illustrated that the working memory is a factor of self-repairs. Speakers who have higher working memory capacity produce lesser self-repairs. This finding provides teachers with a new insight into second language teaching; that is, teachers can improve the amount of lexical knowledge when teaching students who have lower working memory in order to help them produce more accurate language during the process of L2 speech production.
\end{abstract}

Keywords: Self-repair; Working memory capacity

Publication date: August 2021; Online publication: August 30, 2021

\section{Introduction}

Self-repair is considered as an automatic skill of human beings. It connects with one's competence and metalinguistic consciousness ${ }^{[1,2]}$. It provides an important evidence for exploring the speech production process in the field of psycholinguistics ${ }^{[3-5]}$. In addition, Kormos pointed out that speech errors have been considered the most direct evidence for both L1 and L2 speech production in the field of psycholinguistics [5].

For L2, the individual variation was first put forward by Selinger. He explored the influence of the types of self-repairs and concluded that self-repairs are clearly affected by the language background ${ }^{[6]}$. In addition, based on the classification system of Levelt in L1 speech ${ }^{[7]}$, an adaptive taxonomy of self-repairs in L2 speech was presented by Kormos, in which four major groups of L2 self-repairs have been clarified: error, appropriacy, different information, and rephrasing ${ }^{[5]}$. This present study pays attention to selfinitiated repairs. At the same time, the taxonomy by Kormos ${ }^{[5]}$ will be used in the stimulus recall task to calculate the frequency of self-repairs. With respect to exploring the influence on L2 self-repairs, numerous studies have indicated that cognitive mechanisms should be considered to determine individual differences in language comprehension and acquisition. In regard to that, a recent proposal which was presented by Mojavezi and Ahmadian stated that working memory made a new prediction in the influence on the frequency of self-repairs of L2 learners. They assumed that the number and distribution of self-repairs are affected by the working memory capacity ${ }^{[8]}$. Those who have higher working memory capacity usually produce more accurate language and require lesser self-repairs ${ }^{[8]}$; resulting from the fact that working memory has the capacity of controlled attention ${ }^{[9]}$. L2 speakers who possess higher working memory capacity are able to allocate more attention for error detection, which leads to minimal self-repairs ${ }^{[10]}$.

Based on the theoretical framework proposed by Levelt ${ }^{[10]}$, the main purpose of this paper is to explore whether the number of self-repairs is affected by the working memory capacity after calculating the frequency of self-repairs and the scores of working memory. 


\section{Methodology}

A total of 20 Chinese learners of English with high-intermediate level as L2 learners participated in this research. All participants are post-graduate students. They seemed to be of similar English proficiency level, and their IELTS (International English Language Testing System) scores ranged from 6.5 to 7.

In the narrative task, two sets of pictures were selected from the Cambridge Advanced Exam (CAE) sample and the time limit for the speaking task was set to 3 minutes. Each set had three different pictures. These pictures showed different people doing different activities. For example, in the first picture of the first set, five people can be seen climbing a mountain together in an extremely bad winter. Three girls can be seen baking biscuits in the second picture, whereas two boys can be seen as if practicing their band songs in the last picture. Each participant's speech was recorded during the task.

The working memory task had two scores. One of it was the rate of correct judgment of the sentences and the other was the scores for working memory. As the task of judgment in the study was to reduce the influence of adopted strategies in the process of remembering the final words, the rate of correct judgment was not included in the analysis. In the working memory task, participants were asked to listen to a total of 60 sentences in 15 sets, in which each set had a different number of sentences, ranging from two to six sentences. Participants were required to listen to these sets of sentences and judge whether they made sense or not. At the same time, they were also required to recall the final words of each sentence and type them into a textbox, which would appear on the screen at the end of each set. An example is shown as below:

(a) Everybody agrees that middle school can be round for many. (False)

(b) When he was little, my orange brother loved to play with his train set. (False)

(c) The hockey players went to Florida to relax and sunbathe. (True)

(d) Because I wouldn't see him for a long time, it was difficult to say goodbye. (True)

After submission, the participants would know their rate of correct judgment and their final words scores. Additionally, each correct final word had been shown on the screen for them to check their answers. At the end of the task, the overall scores were automatically calculated.

The data in this study were collected by the researcher and all the tasks were carried out by each participant one after another. The instructions for all the tasks were in both Mandarin and English to ensure that the participants clearly understood what they needed to do in this study. As this study is focused on the relationship between self-repair and working memory, it emphasized on the frequency of self-repairs. However, the analyses of specific types of self-repairs were not addressed. Based on the working memory scores, the higher the scores obtained, the participants were assumed to have better memory. All the selfrepairs data in this study were categorized independently.

\section{Results}

The data in this research were analyzed using the Statistical Package for the Social Sciences (SPSS). To begin with, descriptive statistics have been summarized in Figure 1 to show the working memory scores. As illustrated in Figure 1, there is a nominal distribution of the working memory scores $(\mathrm{N}=20$; mean $=$ 34.85). 


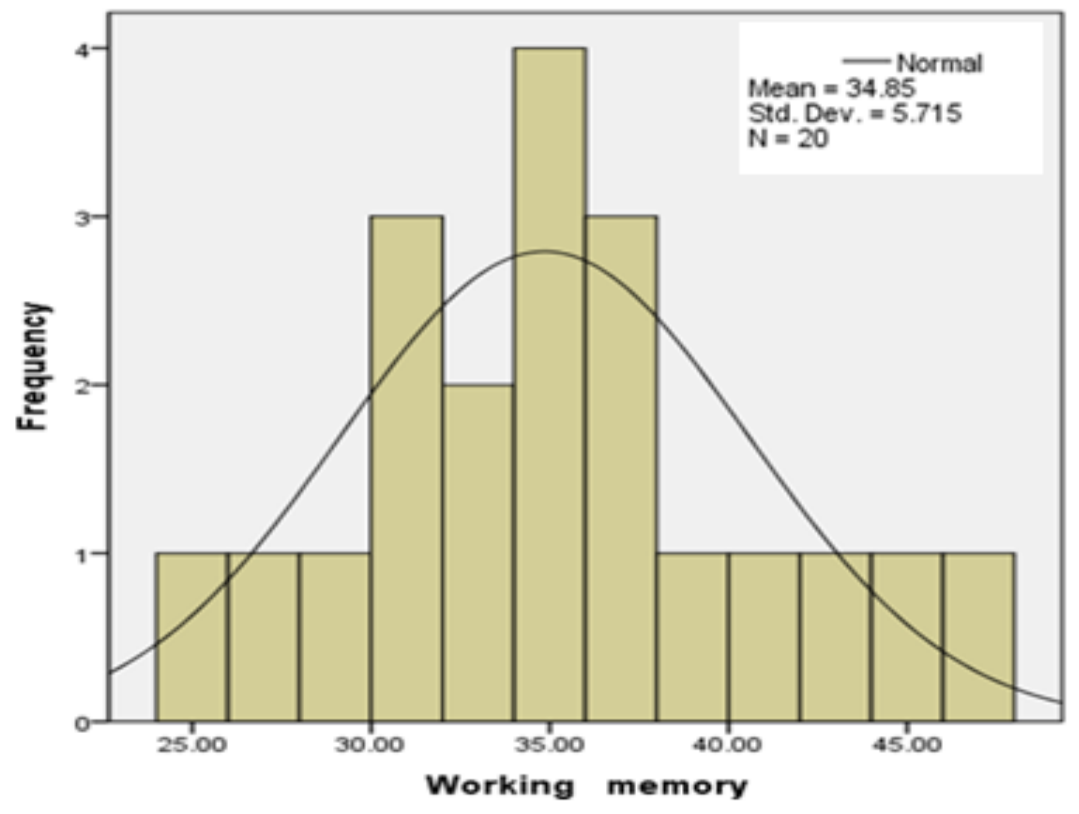

Figure 1. Descriptive statistics of the working memory scores

In view that the data related to the working memory in this study were straight numbers on a continuous scale with a nominal distribution, the ordinary regression method was used to analyze the correlation between the working memory capacity and self-repairs.

A simple regression between the working memory and L2 self-repairs is shown in Figure 2.

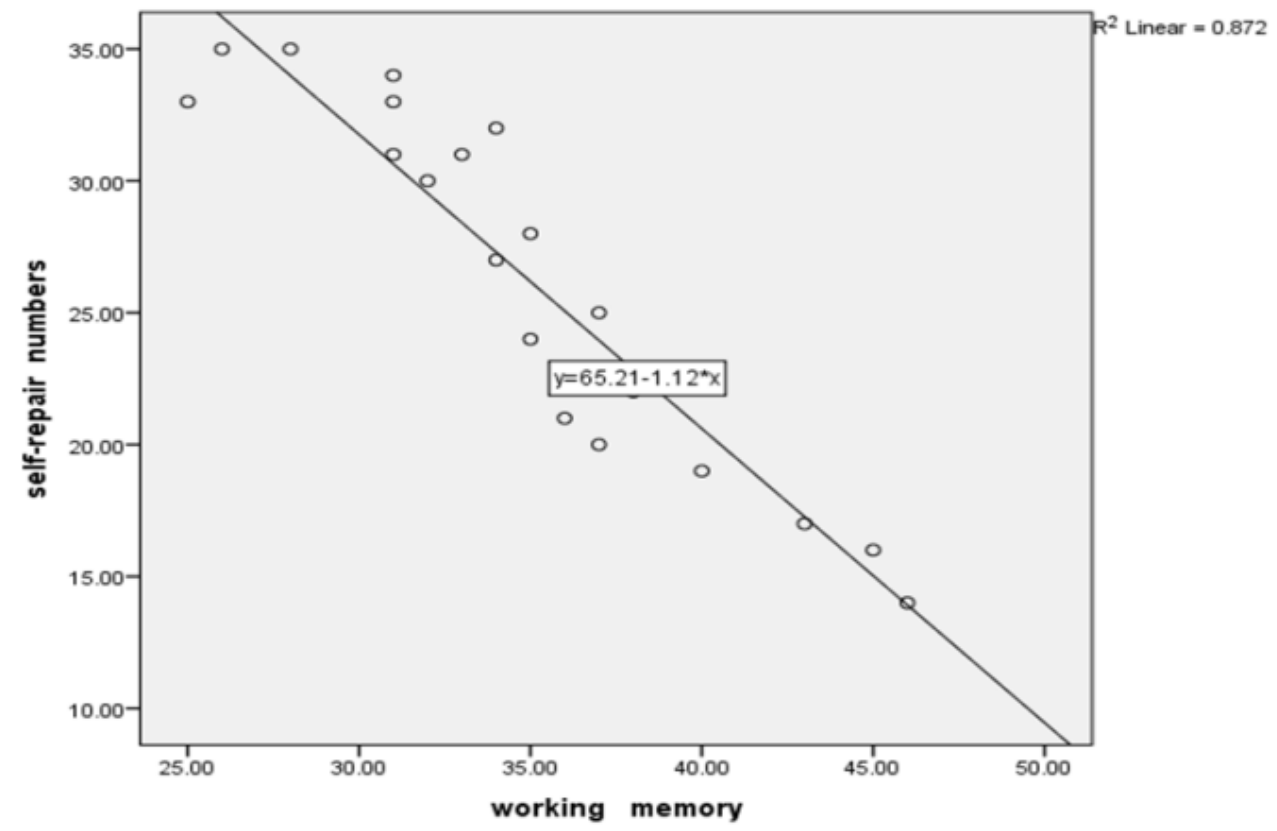

Figure 2. Relationship between working memory capacity and L2 self-repairs

It can be seen in Figure 2 that as the participants' scores on the listening span test increased, their frequency of self-repairs decreased. This means that L2 speakers with higher working memory produced lesser self-repairs. In contrast, L2 speakers with lower working memory produced more self-repairs. This result validates the research question in this study; namely, the working memory capacity might have an impact on the frequency of self-repairs. 


\section{Conclusion}

In order to explore the allocation of attention, Kormos argued that the individual differences in regard to available attention would be affected by the working memory capacity because L2 speakers need to store more lexical messages in their working memory in the process of self-repair ${ }^{[5]}$. Based on this point, the relationship between working memory and self-repairs was analyzed in this study. As a result, the negative correlation between working memory and self-repairs in this study reflects that the influence of working memory on the frequency of self-repairs in L2 learners might be a factor. Temple assumed that the relationship between working memory and L2 language proficiency exists ${ }^{[11]}$, which means that working memory plays an important role in language proficiency, especially for those who have a lower level in the particular language. She stated that it is not always possible for messages to be automatically retrieved from the lexical storage, thus L2 speakers prefer to rely on their working memory in speech production ${ }^{[11]}$. When all of these are considered, it can be concluded that working memory is a factor of self-repairs, and L2 learners with higher working memory capacity produce lesser self-repairs.

From the perspective of psycholinguistics, one explanation for this finding lies in the fact that working memory can be regarded as a source of individual differences ${ }^{[12]}$. As pointed out above, working memory capacity plays an important role in L2 speech production; in addition, it can store and control lexical information according to Levelt ${ }^{[10]}$. It allows speakers to continue monitoring and detecting their speech. Other than that, Fortkamp also claimed that working memory has the capacity of controlled attention ${ }^{[9]}$. With that, speakers can allocate more attention to error detection. Therefore, participants with higher working memory capacity are able to monitor more aspects of their speech and correct their errors, thus producing less self-repairs ${ }^{[10]}$.

The findings from this study provide some practical applications in second language teaching. Considering that working memory is a factor of self-repairs, teachers can improve the amount of lexical knowledge when teaching students who have lower working memory. This would help students to pay more attention to grammatical and lexical errors, thus producing more accurate language. However, there are still some issues. Firstly, a more detailed study should be conducted to explore to what extent the working memory has an influence on language proficiency and thus, affecting self-repairs. Secondly, as it has been pointed out in the literature review section, different types of self-repairs, such as error repair, different information repair, rephrasing repair, and appropriateness repair, should be investigated separately to explore the relationship between working memory and self-repairs in L2 learners. Last but not least, this study focused on only one possible factor of self-repair. However, other variables such as hesitation and the complexity of the task can also be considered. This could provide a new insight to explore L2 selfrepairs. In view of the methodology in this study, since a small group might not provide enough data in regard to self-repairs to reach to a general conclusion, more participants should be recruited to acquire stronger evidence in verifying the influence of working memory capacity on self-repairs to a larger extent.

\section{Disclosure statement}

The author declares that there is no conflict of interest.

\section{References}

[1] Van Hest E, 1996, Self-Repair in L1 and L2 Production, Tilburg University Press, Tilburg. https://www.millisecond.com/download/library/listeningspan/

[2] O'Connor N, 1988, Repairs as Indicative of Interlanguage Variation and Change, Georgetown University Round Table in Languages and Linguistics, 251-9. 
[3] Garrett M, 1975, The Analysis of Sentence Production, in Psychology of Learning and Motivation (Volume 9), Bower G, Academic Press, New York.

[4] Cutler A, 2011, Slips of the Tongue and Language Production, Walter de Gruyter.

[5] Kormos J, 1999, Monitoring and Self-Repair in L2. Language Learning, 49(2): 303-42.

[6] Selinger H, 1980, Utterance Planning and Correction Behavior: Its Function in the Grammar Construction Process for Second Language Learners, in Towards a Cross-Linguistic Assessment of Speech Production, Dechert H, Raupach M, Lang, Cirencester.

[7] Levelt WJ, 1983, Monitoring and Self-Repair in Speech. Cognition, 14: 41-104.

[8] Mojavezi A, Ahmadian MJ, 2014, Working Memory Capacity and Self-Repair Behavior in First and Second Language Oral Production. Journal of Psycholinguistic Research, 43(3): 289-97.

[9] Fortkamp MBM, 2000, Working Memory Capacity and L2 Speech Production: An Exploration Study.

[10] Levelt WJ, 1989, Speaking: From Intention to Articulation, MIT press, Cambridge.

[11] Temple L, 1997, Memory and Processing Modes in Language Learner Speech Production. Communication and Cognition, 30(1): 75-90.

[12] Daneman M, Carpenter PA, 1980, Individual Differences in Working Memory and Reading. Journal of Verbal Learning and Verbal Behavior, 19(4): 450-66. 